\title{
Article
}

\section{Hypoxic Exercise Training Elevates Erythrocyte Aggregation}

\author{
Chin-Li Lin ${ }^{1,2,+}$, Jong-Shyan Wang $2,3,4,+$, Tieh-Cheng Fu ${ }^{3}$, Chih-Chin Hsu ${ }^{3} \mathbb{D}$ and Yu-Chieh Huang ${ }^{5, * \mathbb{D}}$ \\ 1 Department of Athletics Training and Health, College of Exercise and Health Sciences, National Taiwan Sport \\ University, Taoyuan 333, Taiwan; king@ntsu.edu.tw \\ 2 Healthy Aging Research Center, Graduate Institute of Rehabilitation Science, Medical College, Chang Gung \\ University, Taoyuan 333, Taiwan; s5492@mail.cgu.edu.tw \\ 3 Heart Failure Center, Department of Physical Medicine and Rehabilitation, Chang Gung Memorial Hospital, \\ Keelung 204, Taiwan; fic6481@gmail.com (T.-C.F.); steele0618@gmail.com (C.-C.H.) \\ 4 Research Center for Chinese Herbal Medicine, College of Human Ecology, Chang Gung University of Science \\ and Technology, Taoyuan 333, Taiwan \\ 5 Department of Physical Therapy, College of Medical and Health Science, Asia University, \\ Taichung 413, Taiwan \\ * Correspondence: yuchieh@asia.edu.tw \\ + These authors contributed equally to this work.
}

Citation: Lin, C.-L.; Wang, J.-S.; Fu, T.-C.; Hsu, C.-C.; Huang, Y.-C. Hypoxic Exercise Training Elevates Erythrocyte Aggregation. Appl. Sci. 2021, 11, 6038. https://doi.org/ 10.3390/app11136038

Academic Editor: Shin-Da Lee

Received: 11 May 2021

Accepted: 26 June 2021

Published: 29 June 2021

Publisher's Note: MDPI stays neutral with regard to jurisdictional claims in published maps and institutional affiliations.

Copyright: (c) 2021 by the authors. Licensee MDPI, Basel, Switzerland. This article is an open access article distributed under the terms and conditions of the Creative Commons Attribution (CC BY) license (https:// creativecommons.org/licenses/by/ $4.0 /)$.

\begin{abstract}
Pathological erythrocyte aggregation reduces capillary perfusion and oxygen transfer to tissue, which is determined by the negative surface charge on the erythrocyte membrane (intrinsic aggregability) and fibrinogen-erythrocyte interaction (extrinsic factor). Exercise-induced oxidative stress is important for rheological adaptation to training but may also cause erythrocyte senescence. This study clarifies the effects of hypoxic exercise training on intrinsic/extrinsic factors of aggregation. In total, 60 healthy sedentary males were randomly assigned to either hypoxic (HE; $\mathrm{F}_{\mathrm{I}} \mathrm{O}_{2}=0.15$ ) or normoxic exercise training $\left(\mathrm{NE} ; \mathrm{F}_{\mathrm{I}} \mathrm{O}_{2}=0.21\right)$ groups for $30 \mathrm{~min} \cdot \mathrm{d}^{-1}, 5 \mathrm{~d} \cdot \mathrm{wk}^{-1}$ for 6 weeks at $60 \%$ of the maximum work rate or to a control group (CTL). A hypoxia exercise test (HET, $\mathrm{F}_{\mathrm{I}} \mathrm{O}_{2}=0.12$ ) was performed before and after the intervention. Erythrocyte aggregation was assessed by ektacytometry, and fibrinogen binding affinity and senescence biomarkers were assessed by flow cytometry. An acute $12 \%$ oxygen HET significantly enhanced erythrocyte global aggregation through intrinsic aggregability. Resting aggregation is promoted by both intrinsic aggregability and fibrinogen binding probability and force after HE, whereas NE is mainly associated with ameliorated fibrinogen-erythrocyte interactions. The HET still facilitated global aggregation after HE because of the augmented fibrinogen-related factors, even though the intrinsic factor was suppressed. Additionally, HE further increased reticulocyte counts while reducing the expression of CD47 and CD147. Resting aggregability is promoted by both intrinsic and extrinsic factors after HE, whereas NE is mainly associated with an ameliorated affinity for fibrinogen. Although an accelerated turnover rate was observed, HE further led to erythrocyte senescence.
\end{abstract}

Keywords: erythrocyte; aggregation; hypoxia; exercise training; fibrinogen

\section{Introduction}

Erythrocyte aggregation is a critical hemorheological property that contributes to dynamically regulating blood flow and influencing oxygen delivery. Impaired rheological functions of erythrocytes lead to circulatory disorders, such as myocardial ischemia and infarction [1], cerebral infarction [2], and diabetes mellitus [3]. Erythrocyte aggregation occurs at low shear rates and depends on both cellular (intrinsic erythrocyte aggregability) and plasma fibrinogen-related erythrocyte adhesion factors (extrinsic factors) [4], as well as the disaggregating shear force generated by blood flow [3]. A great amount of evidence indicates that the aggregation tendency can be modified by several properties, such as erythrocyte aging in vivo [5], reactive oxygen species (ROS) [6], inflammatory status [7], or the blood hematocrit [8]. 
Endurance exercise is an efficient and safe strategy to improve cardiopulmonary capacity. A number of studies have well established the effects of endurance training on hemorheological properties [9]. In patients with marked obesity or insulin resistance who receive therapeutic exercise training, plasma viscosity and hemorheology are mostly improved and reflect a change in plasma fibrinogen [10]. Altitude/hypoxic training appears to be more effective than sea-level/normoxic training for improving cardiopulmonary capacity [11]. However, exposure to a hypoxic environment may incur a progressive rigidity of erythrocytes [12] or even deteriorate antioxidative capacity [13]. Of clinical relevance, inappropriate hypoxia, such as severe sleep apnea, impairs rheological properties as well [12].

Elevated erythropoietin (EPO) production in hypoxia is a key factor in the achievement of enhanced hematological variables. Its level depends on the duration of exposure and degree of hypoxia [14]. Hypoxic exercise markedly lowers the oxygen concentration or saturation of working organs by simultaneously decreasing oxygen supply and increasing oxygen demand to augment hypoxemia. Therefore, hypoxic exercise training may trigger hypoxia-inducible factor-1 (HIF-1)-mediated processes, then stimulate EPO secretion [15]. Despite an elevated plasma EPO level, the repeated, excessive exposure to ROS may further trigger eryptosis or neocytolysis, thereby counterbalancing erythropoiesis induced by EPO or accumulating stress reticulocytes [16].

Our previous study demonstrated that hypoxic exercise training (HE) causes erythrocyte senescence and simultaneously promotes eryptotic response to oxidative stress [13]. In addition, intermittent hypoxia exposure, acute exercise, or inflammation status promotes platelet aggregation [17] and also elevates the plasma fibrinogen concentration [14]. Therefore, excessive oxidative stress is caused by acute hypoxia exercise, which may trigger eryptosis and lead to surface charge alteration, whereas chronic hypoxia exposure is speculated to enhance erythrocyte affinity with fibrinogen [18]. The effects of hypoxic exercise training on the erythrocyte aging process, eryptotic response to oxidative stress, and aggregation properties remain unclear [19]. We hypothesize that hypoxic exercise training impairs erythrocyte rheological functions by raising affinity to fibrinogen and suppressing the negative charge via facilitating erythrocyte senescence and turnover rate.

The aim of this study was to clarify the distinct effects of exercise training with or without hypoxia on erythrocyte aggregation, further providing a feasible strategy for developing an appropriate exercise regimen that improves cardiopulmonary fitness and minimizes the risk of hemorheological disorders. This study evaluated the following issues: (1) intrinsic aggregability, (2) fibrinogen binding probability and force, (3) mature erythrocyte and reticulocyte subfraction, and (4) erythrocyte senescence marker expression during a hypoxic exercise test (HET) in healthy sedentary men.

\section{Materials and Methods}

\subsection{Subjects}

This investigation followed the Declaration of Helsinki and was approved by the Institutional Review Board of Chang Gung Memorial Hospital in Taiwan (200903510B0), and it was published on ClinicalTrials.gov (NCT04805359). The study population included 60 sedentary males who were nonsmokers, did not take any medications or vitamins, did not have any cardiopulmonary/hematological risk, had a sedentary lifestyle (without regular exercise, exercise frequency of at most once weekly, for a duration of $<20 \mathrm{~min}$ ), and had been exposed to high altitudes $(>3000 \mathrm{~m})$ for at least 1 year. All subjects provided informed consent after the experimental procedures were explained. All subjects were randomly divided into three groups: the HE $(n=20), \mathrm{NE}(n=20)$, and control (CTL) groups. All subjects arrived at the testing center at 9:00 a.m. to eliminate any possible circadian effects and were instructed to fast for at least $8 \mathrm{~h}$ and to refrain from strenuous physical exercise for at least $48 \mathrm{~h}$ before sampling. 


\subsection{Protocol and Interventions}

Both the HE and NE groups performed exercise regimens on a stationary bicycle ergometer (Corival 400, Lode B.V., Groningen, The Netherlands) 5 times a week for 6 weeks in an air-conditioned normobaric hypoxia chamber (Figure 1). For comparison, those in the CTL group did not undertake any exercise and were carefully monitored and recorded in terms of physical activity and daily diet. Both intervention groups were warmed up for $3 \mathrm{~min}$ at $30 \%$ of the maximal work rate $\left(\mathrm{WR}_{\max }\right)$, followed by $30 \mathrm{~min}$ of $60 \% \mathrm{WR}_{\max }$ cycling under $15 \% \mathrm{O}_{2}$ (HE) or $21 \% \mathrm{O}_{2}$ (NE) conditions. Oxygen concentrations of 15 and $21 \%$ correspond to altitudes of $\approx 2733 \mathrm{~m}$ and sea level, respectively. The hypoxia chamber was maintained at a temperature of $22 \pm 0.5{ }^{\circ} \mathrm{C}$ and relative humidity of $60 \pm 5 \%$; a $\mathrm{CO}_{2}$ scrubber eliminated $\mathrm{CO}_{2}$ from the air ( $<3500$ ppm; Colorado Mountain Room). All groups were asked to record their daily activities and nutritional intake by using the International Physical Activity Questionnaire Short Form [20] and the Written Diet Record [21], respectively. Subjects were asked not to perform any extra regular exercise until the end of the study. Moreover, all subjects completed the experiment, and the participant compliance rate was $100 \%$.

\subsection{Cardiopulmonary Exercise Test (CPET)}

To assess cardiopulmonary fitness, a cardiopulmonary exercise test (CPET) on a cycle ergometer was performed 4 days before and after the intervention. All subjects underwent exercise with a facemask fixed on the face to measure minute ventilation $\left(\dot{\mathrm{V}}_{\mathrm{E}}\right)$, oxygen consumption $\left(\dot{\mathrm{VO}}_{2}\right)$, and carbonic dioxide production $\left(\dot{\mathrm{V}} \mathrm{CO}_{2}\right)$ breath by breath using a computer-based system (Master Screen CPX, CareFusion, Höchberg, Germany). After $5 \mathrm{~min}$ baseline resting period records, a $2 \mathrm{~min}$ warm-up period (60 rpm, unloaded pedaling) was started, followed by incremental work ( $30 \mathrm{~W}$ elevation for each $3 \mathrm{~min}$ ) until exhaustion (i.e., progressive exercise to maximal $\mathrm{O}_{2}$ consumption, $\dot{\mathrm{V}}_{2 \max }$ ). The criteria used to define $\mathrm{VO}_{2 \max }$ and the ventilation threshold were consistent with the guidelines of the American College of Sports Medicine for exercise testing [22]. During the CPET, continuous monitoring of 12-lead electrocardiography, blood pressure, and pulse oxygen saturation was performed. Oxygen saturation $\left(\mathrm{SpO}_{2}\right)$ was monitored by finger pulse oximetry (BCI 3301, Smiths Medical PM, Inc., Waukesha, WI, USA); the blood lactate concentration was determined using an i-STAT clinical analyzer (i-STAT analyzer, Abbott, Chicago, IL, USA).

\subsection{Hypoxia Exercise Test (HET)}

Each subject performed the hypoxia exercise test (HET) 2 days before and after the intervention in a $12 \%$ oxygen-concentration-controlled normobaric hypoxia chamber (Colorado Mountain Room, Boulder, CO, USA), as described in previous studies [23]. The HET protocol contained a 5 min warm-up at $30 \% \mathrm{WR}_{\max }, 30 \mathrm{~min}$ at $60 \% \mathrm{WR}_{\max }$, and a 5 min cool-down at $0 \% \mathrm{WR}_{\max }$. For safety, the HET would terminate immediately if the $\mathrm{SaO}_{2}$ dropped below $70 \%$. All subjects maintained their $\mathrm{SaO}_{2}$ above $70 \%$ during the entire exercise period in this study.

\subsection{Erythrocyte Isolation and Blood Collection}

At rest and immediately after the HET, a $10 \mathrm{~mL}$ blood sample was collected from the antecubital vein via clean venipuncture (21-gauge needle) and added to a tube with sodium citrate $(0.106 \mathrm{M})$. Blood cells were counted by using a Sysmax SF-3000 cell counter (GMI Inc., Ramsey, MN, USA) [6]. Erythrocytes were isolated from whole blood by centrifugation $(1000 \times g$ for $15 \mathrm{~min}$ at RT), the supernatant was discarded, and the buffy coat was discarded, followed by three washing steps with phosphate-buffered saline. 
(A)

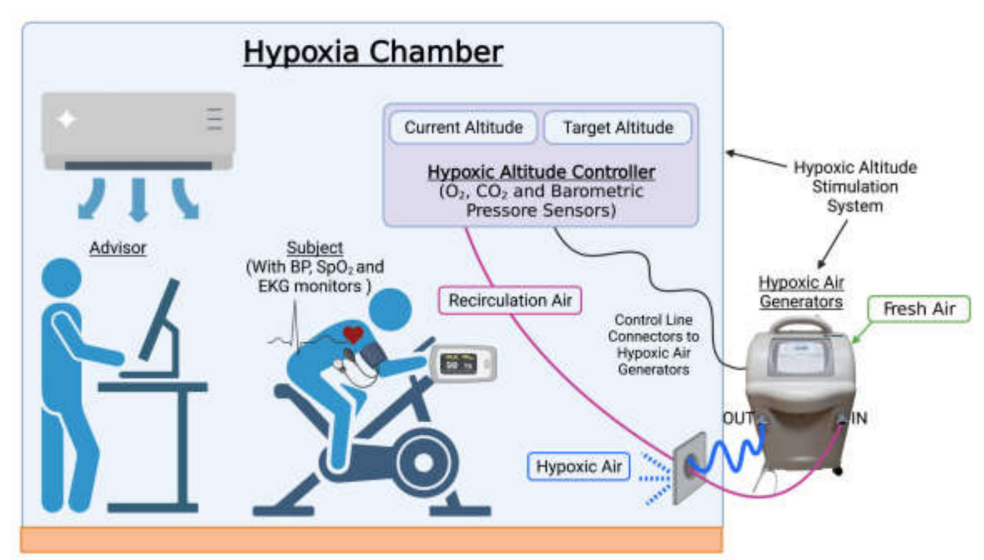

(B)
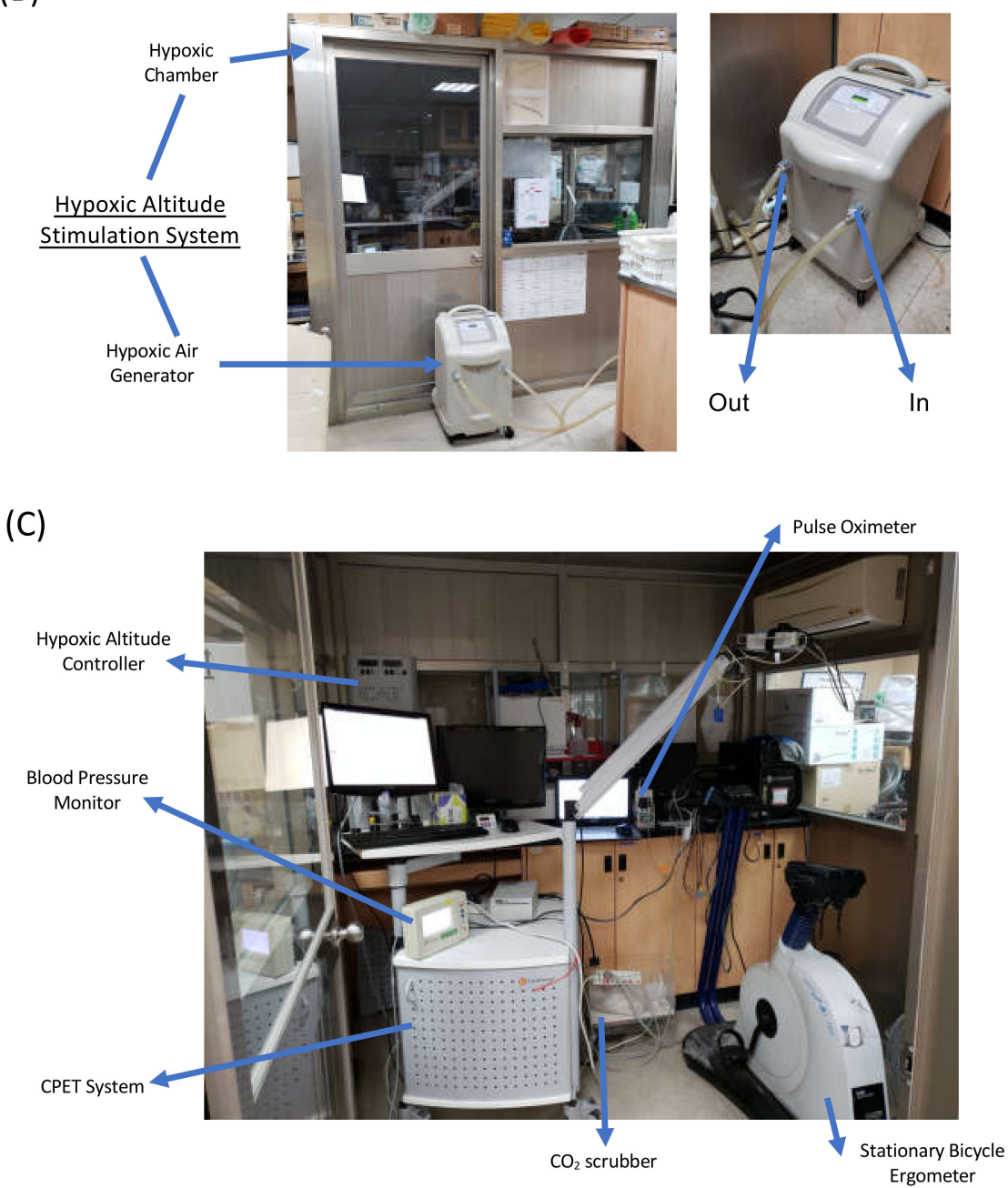

Figure 1. Experimental setup: (A) schematic representation of hypoxia chamber setup; running hypoxia experiment: $(\mathbf{B})$ the outside of the chamber; $(\mathbf{C})$ the inside layout of the chamber.

\subsection{Erythrocyte Aggregation}

Aggregation was determined by using a microfluidic Ektacytometer (Rheoscan-AnD 300, RheoMeditech, Seoul, Korea). The blood sample was added to a microchannel kit as the user manual described and driven by differential pressure through the narrow microchannel. When a high differential pressure is applied, strong shear stress occurs 
along the microchannel, and the erythrocytes start to disaggregate. Following continuously decreasing pressure differentials, erythrocyte aggregates tend to disperse at high shear flows, and the corresponding backscattered light (BSL) intensity increases. As the pressure differential decreases further, the dispersed erythrocytes reaggregate, and the BSL intensity decreases. The time and shear stress corresponding to the maximal BSL intensity were defined as the critical time (CT) and the critical shear stress (CSS), respectively.

To measure global blood aggregation, whole blood $(500 \mu \mathrm{L})$ was added into the reservoir chamber without adjusting for Hct. Furthermore, in order to eliminate the dehydration effect after exercise (hemoconcentration), the Hct was adjusted to $40 \%$ before treating the erythrocyte isolation with $400 \mathrm{mM}$ fibrinogen (Sigma) or dextran (10 mM, Sigma).

\subsection{The Affinity between Erythrocytes and Fibrinogen}

Erythrocyte suspension $\left(1 \times 10^{6}\right.$ cells $\left./ \mu \mathrm{L}\right)$ was incubated with fibrinogen $(400 \mathrm{mM})$ and anti-fibrinogen-FITC monoclonal antibody (1:500, Cedarlane) in the dark for $30 \mathrm{~min}$ at RT. After the staining was completed, $1 \mu \mathrm{L}$ suspension was added to $100 \mu \mathrm{L}$ medium solution, and then the sample was rotated on a viscometer (DV1, Brookfield, Middleboro, MA, USA) at different rates $(0,50,100,200,400,800 \mathrm{rpm})$ for $1 \mathrm{~min}$ at $37^{\circ} \mathrm{C}$ in the dark. The sample was quickly added to the fixation buffer in the dark for 10 min at RT. The mean fluorescence intensity obtained from 50,000 erythrocytes was measured by a FACSalibur (Becton Dickinson, NJ, USA).

\subsection{Reticulocyte Counts}

Erythrocyte suspension $\left(1 \times 10^{6}\right.$ cells $\left./ \mu \mathrm{L}\right)$ was incubated with anti-human CD71 antibody conjugated with phycoerythrin (DB) and thiazole orange (TO), an RNA-binding fluorochrome dye (Sigma-Aldrich), in the dark for $30 \mathrm{~min}$ at $37^{\circ} \mathrm{C}$, then washed twice with Hank's balanced salt solution (HBSS). The relative distribution of three different erythrocyte subfractions was determined according to the expression of $\mathrm{CD}^{+/-}$and $\mathrm{TO}^{+/-}$. Stained $\mathrm{CD}^{+} 1^{+} / \mathrm{TO}^{+}$indicated reticulocytes, expressed $\mathrm{CD} 71^{+} / \mathrm{TO}^{-}$or $\mathrm{CD} 71^{-} / \mathrm{TO}^{+}$represented stress reticulocytes [16], and $\mathrm{CD}^{-} / \mathrm{TO}^{-}$indicated mature erythrocytes [24]. The fraction obtained from a total of 50,000 cells was measured by a FACSCalibur (Becton Dickinson, New Jersey, USA) [25].

\subsection{Adhesion- and Senescence-Related Molecules on Erythrocytes}

Erythrocyte suspensions $\left(1 \times 10^{6}\right.$ cells $\left./ \mu \mathrm{L}\right)$ were incubated with monoclonal antiCD147 (eBioscience), anti-CD47 (BioLegend), anti-CD49d (eBioscience), and anti-CD36 (BioLegend) antibodies conjugated with FITC in the dark for $30 \mathrm{~min}$ at $37^{\circ} \mathrm{C}$. The mean fluorescence intensity obtained from 50,000 erythrocytes was measured using a FACSCalibur.

\subsection{Statistical Analysis}

The results are expressed as means \pm SEM. The statistical software SPSS 22.0 (SPSS, Chicago, IL, USA) was used for data analysis. Experimental results were analyzed by a 3 (groups) $\times 4$ (time sample points) repeated-measures ANOVA and Bonferroni's post hoc test to compare reticulocyte and erythrocyte counts, erythrocyte aggregation, and the affinity of fibrinogen before and immediately after HET at the beginning of the present study and after 6 weeks in various interventions. In addition, comparisons of cardiopulmonary fitness during the CPET at the beginning of the present study and 6 weeks later in the various groups were analyzed by a 3 (groups) $\times 4$ (time sample points) repeated-measures ANOVA and Bonferroni's post hoc test. The criterion for significance was $p<0.05$.

\section{Results}

\subsection{Cardiopulmonary Capacity}

None of the three groups differed significantly in their anthropometric parameters or exercise performance at the beginning of the study (Table 1). Following six weeks of interventions, both $\mathrm{NE}$ and $\mathrm{HE}$ significantly increased the work rate, $\dot{\mathrm{V}}_{\mathrm{E}}$, and $\dot{\mathrm{V}}_{2}$ at the 
ventilatory threshold and peak exercise performance; furthermore, HE was superior to NE in improving the peak performance (Table $1, p<0.05$ ). In addition, no significant differences in cardiopulmonary responses to the CPET were observed after 6 weeks of CTL (Table 1).

Table 1. Anthropometric data and cardiopulmonary exercise test (normoxia) performance in NE and HE.

\begin{tabular}{|c|c|c|c|c|c|c|}
\hline & \multicolumn{2}{|c|}{$\mathrm{NE}$} & \multicolumn{2}{|c|}{ HE } & \multicolumn{2}{|c|}{ CTL } \\
\hline & Pretraining & Post-Training & Pretraining & Post-Training & Pretraining & Post-Training \\
\hline \multicolumn{7}{|c|}{ Anthropometric variables } \\
\hline Age (years) & $22.4 \pm 0.9$ & - & $21.9 \pm 0.7$ & - & $23.1 \pm 0.9$ & - \\
\hline Height (cm) & $173 \pm 2$ & - & $173 \pm 3$ & - & $174 \pm 2$ & - \\
\hline Weight $(\mathrm{kg})$ & $68.1 \pm 2.2$ & $66.9 \pm 3.1$ & $66.9 \pm 2.5$ & $66.7 \pm 2.3$ & $65.7 \pm 2.3$ & $66.2 \pm 1.8$ \\
\hline \multicolumn{7}{|c|}{ Ventilation threshold performance } \\
\hline Work Rate (watt) & $121 \pm 7$ & $155 \pm 7$ * & $123 \pm 4$ & $156 \pm 4^{*}$ & $122 \pm 5$ & $124 \pm 4$ \\
\hline$\dot{\mathrm{V}}_{\mathrm{E}}\left(\mathrm{L} \min ^{-1}\right)$ & $55.5 \pm 3.6$ & $72.0 \pm 4.6 *$ & $55.8 \pm 4.5$ & $70.4 \pm 4.3 *$ & $55.6 \pm 4.1$ & $56.3 \pm 4.2$ \\
\hline$\dot{\mathrm{VO}}_{2}\left(\mathrm{~mL} \mathrm{~kg}^{-1} \mathrm{~min}^{-1}\right)$ & $23.7 \pm 1.3$ & $30.0 \pm 1.8^{*}$ & $23.9 \pm 1.7$ & $28.2 \pm 1.3 *$ & $23.4 \pm 1.6$ & $23.2 \pm 1.5$ \\
\hline $\mathrm{HR}(\mathrm{bpm})$ & $149 \pm 3$ & $157 \pm 3 *$ & $147 \pm 4$ & $153 \pm 3$ & $147 \pm 4$ & $149 \pm 3$ \\
\hline $\mathrm{CO}\left(\mathrm{L} \mathrm{min}^{-1}\right)$ & $14.3 \pm 0.6$ & $15.1 \pm 0.6$ & $14.4 \pm 0.5$ & $15.6 \pm 0.8$ & $14.1 \pm 0.4$ & $14.4 \pm 0.6$ \\
\hline MAP (mmHg) & $119 \pm 2$ & $123 \pm 3$ & $117 \pm 2$ & $118 \pm 2$ & $116 \pm 3$ & $115 \pm 2$ \\
\hline \multicolumn{7}{|l|}{ Peak performance } \\
\hline$\overline{\text { Work Rate (watt) }}$ & $187 \pm 5$ & $216 \pm 5^{*}$ & $189 \pm 5$ & $228 \pm *,+$ & $188 \pm 5$ & $189 \pm 5$ \\
\hline$\dot{\mathrm{V}}_{\mathrm{E}}\left(\mathrm{L} \min ^{-1}\right)$ & $117.9 \pm 3.4$ & $124.4 \pm 3.9$ * & $117.7 \pm 4.8$ & $137.9 \pm 3.6^{*,+}$ & $116.86 \pm 3.9$ & $117.9 \pm 3.1$ \\
\hline$\dot{\mathrm{VO}}_{2}\left(\mathrm{ml} \mathrm{kg}^{-1} \min ^{-1}\right)$ & $33.6 \pm 1.1$ & $36.3 \pm 1.1 *$ & $33.4 \pm 1.4$ & $38.8 \pm 1.3^{*,+}$ & $33.8 \pm 1.3$ & $32.2 \pm 1.1$ \\
\hline $\mathrm{HR}(\mathrm{bpm})$ & $195 \pm 2$ & $193 \pm 2$ & $193 \pm 2$ & $193 \pm 2$ & $193 \pm 2$ & $194 \pm 3$ \\
\hline $\mathrm{CO}\left(\mathrm{L} \mathrm{min}^{-1}\right)$ & $16.0 \pm 0.5$ & $16.3 \pm 0.4$ & $16.3 \pm 0.6$ & $18 \pm 0.5 *$ & $16.2 \pm 0.4$ & $15.9 \pm 0.5$ \\
\hline MAP (mmHg) & $128 \pm 2$ & $133 \pm 3$ & $129 \pm 2$ & $132 \pm 2$ & $129 \pm 2$ & $128 \pm 3$ \\
\hline
\end{tabular}

Values are mean $\pm \mathrm{SEM}$. $\dot{\mathrm{V}}_{\mathrm{E}}$, minute ventilation; $\dot{\mathrm{VO}}_{2}$, oxygen consumption; HR, heart rate; $\mathrm{CO}$, cardiac output; MAP, mean arterial pressure; NE, normoxia training group; HE, hypoxia training group; CTL, control group. ${ }^{*} p<0.05$, Pre vs. Post; ${ }^{\dagger} p<0.05$, NE vs. HE.

\subsection{Hypoxia Exercise Test Performance}

Acute HET significantly decreased the $\mathrm{SpO}_{2}$, blood $\mathrm{pH}$ value, and $\mathrm{PCO}_{2}$ and increased the blood lactate concentration, erythrocyte count, hemoglobin, hematocrit, and EPO. Both $\mathrm{NE}$ and HE for 6 weeks significantly diminished the exercise-related augmentation of the HR, $\mathrm{pH}$ value, and Lac concentration, which indicated improved anti-hypoxic stress capacity under the same hypoxic challenge. No alterations in the erythrocyte count, hemoglobin level, or hematocrit were found in NE or HE, whereas EPO was significantly augmented after HE (Table 2).

Table 2. Effects of NE and HE on erythrocyte characteristics and serum erythropoietin concentration in HET (hypoxia).

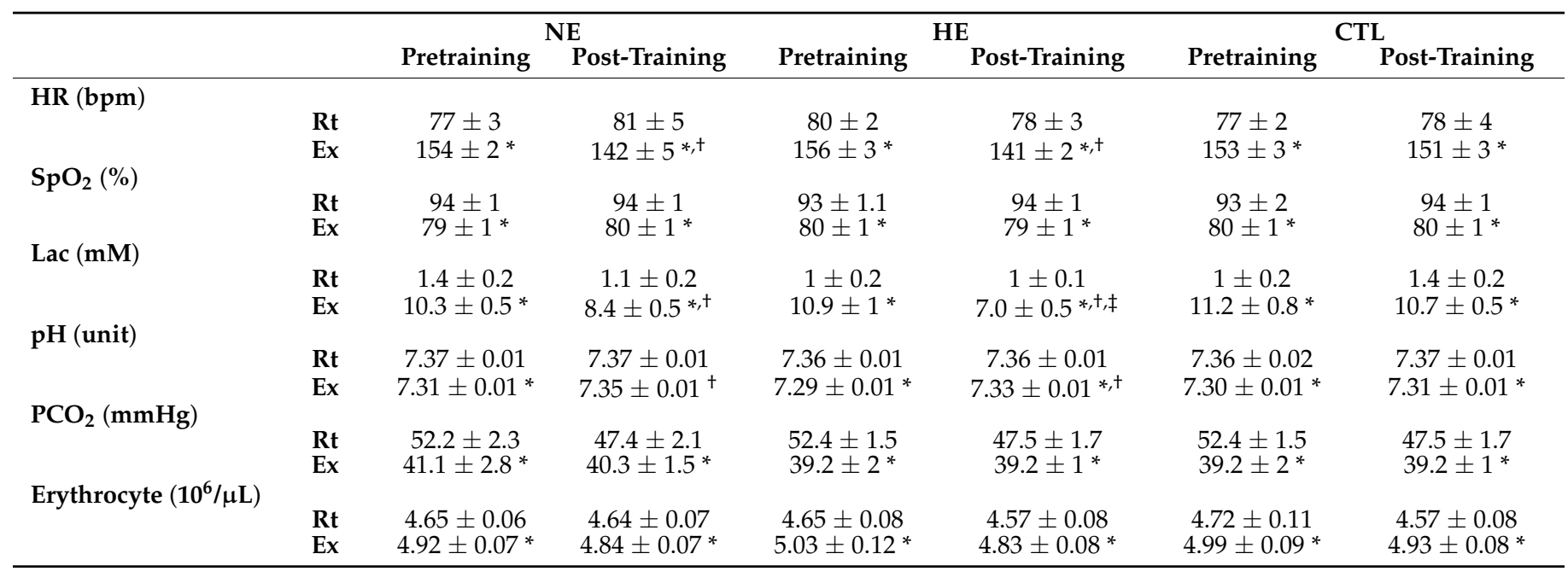


Table 2. Cont.

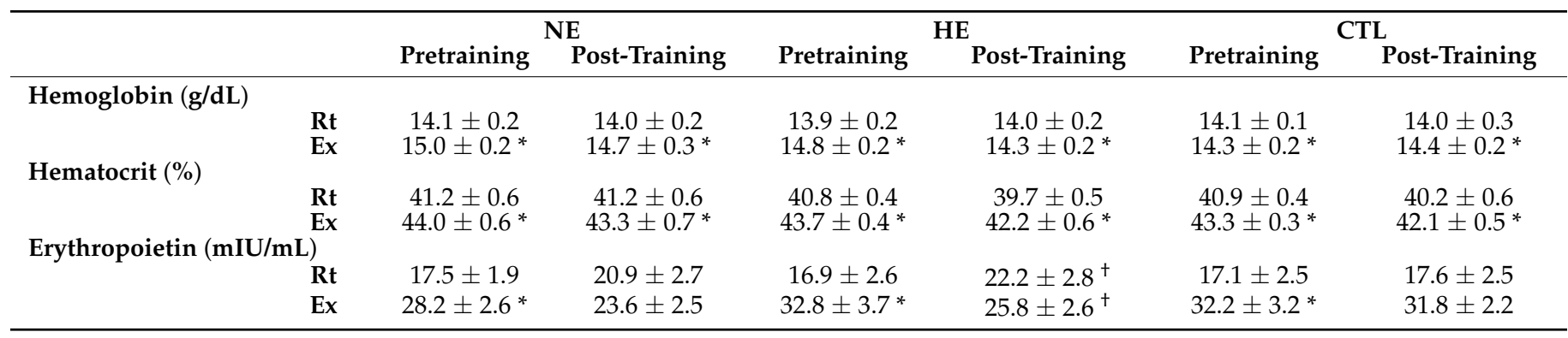

Values are the mean \pm SEM. NE, normoxia training group; HE, hypoxia training group; CTL, control group; HET, hypoxia exercise test; $\mathrm{HR}$, heart rate; $\mathrm{SpO}_{2}$, pulse oxygen saturation; $\mathrm{PCO}_{2}$, the partial pressure of carbon dioxide; Lac, blood lactate concentration; Rt, resting before the HET; Ex, immediately after the HET. ${ }^{*} p<0.05$, Rt vs. Ex; ${ }^{\dagger} p<0.05$, pretraining vs. post-training; ${ }^{\ddagger} p<0.05, \mathrm{HE}$ vs. NE.

\subsection{Whole Blood Aggregation}

The whole blood aggregation responses of the various training regimens are shown in Figure 2A,B. As the data show, acute HET induced augmented global aggregation among the three groups. In contrast to NE, HE significantly enhanced resting aggregation after 6 weeks (Figure 2A,B, $p<0.05$ ).
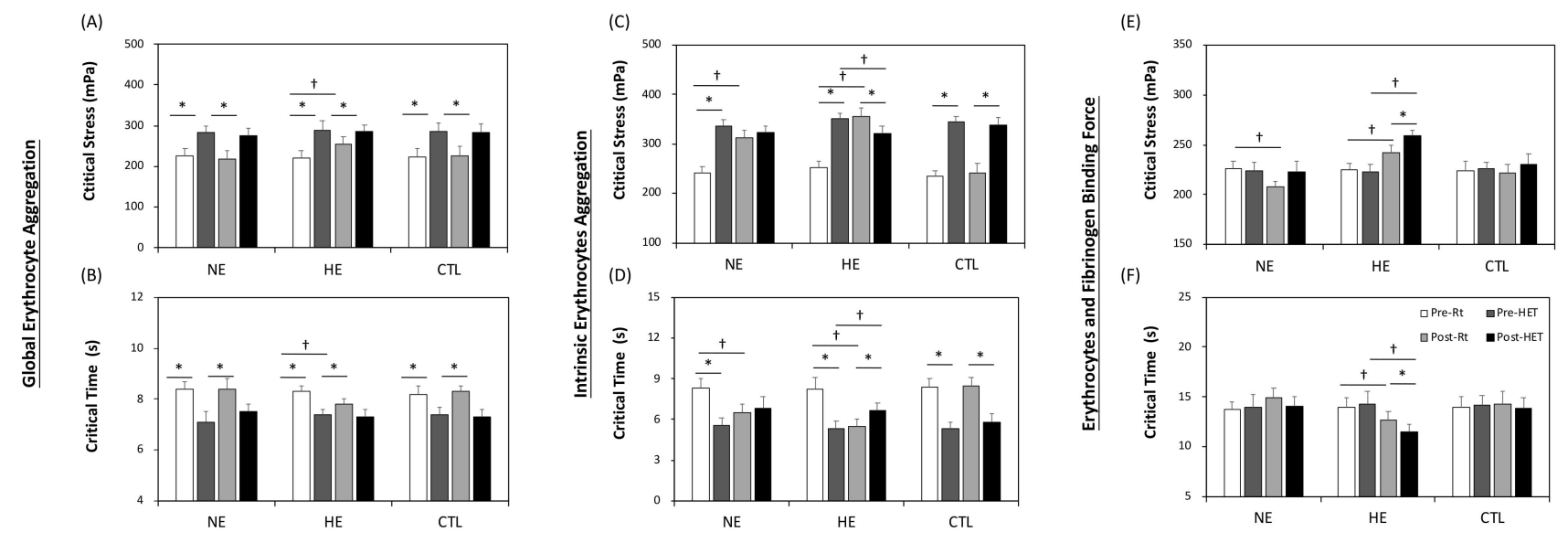

Figure 2. Measurement of erythrocyte aggregation. Global erythrocyte aggregation without hematocrit adjustment: (A) critical stress; (B) critical time. The repulsive force between the negatively charged cells (an intrinsic factor): (C) critical stress; (D) critical time. Cell-to-cell aggregation induced by fibrinogen (an extrinsic factor): (E) critical stress; (F) critical time. $\mathrm{NE}$, normoxia training group; HE, hypoxia training group; CTL, control group; Pre, pretraining; Post, post-training; Rt, at rest; HET, immediately after HET; ${ }^{*} p<0.05$, Rt vs. HET; $\uparrow p<0.05$, pre vs. post. Values are the mean $\pm \mathrm{SEM}$.

\subsection{Intrinsic Factor of Aggregation}

To concentrate on investigating the repulsive force between the negatively charged cells, dextran was added to rule out any environmental influence. According to Figure 2C,D, intrinsic aggregation was enhanced by acute hypoxic stress; furthermore, the influence was augmented by both NE and HE. Nevertheless, unlike NE, HE exhibited resistance under hypoxic stress, as indicated by the lower critical stress after HET.

\subsection{Fibrinogen (Extrinsic) Factor of Aggregation}

The results related to the cell-to-cell adhesion induced by fibrinogen are displayed in Figure 2E,F. No change in the binding force affected by acute hypoxic stress was observed. However, NE and HE had the opposite response after 6 weeks of training. A reduced fibrinogen-to-cell binding probability was noticed in NE, whereas HE dramatically enhanced the binding percentage, representing a higher fibrinogen affinity than NE (Figure 3). 
(A)

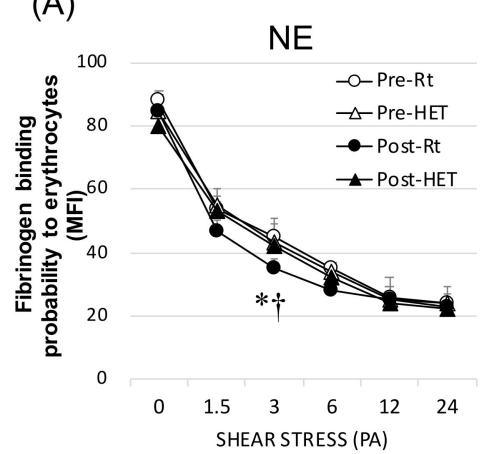

(B)

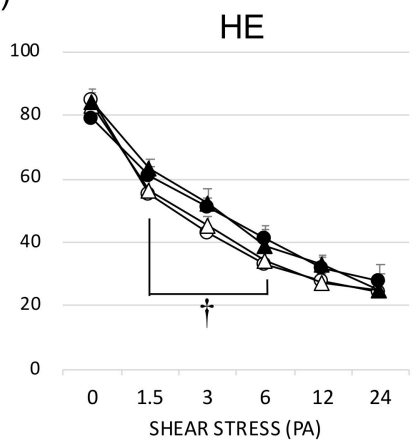

(C)

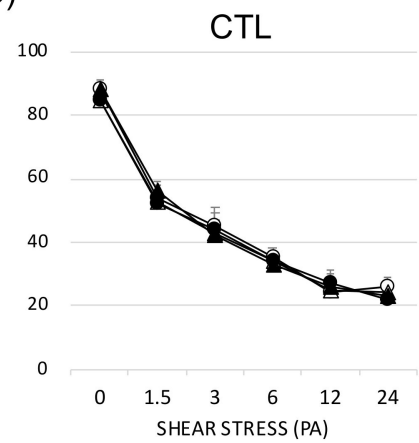

Figure 3. Measurement of fibrinogen binding probability to erythrocytes: (A) normoxia training group, (B) hypoxia training group, and (C) control group. Pre (white), pretraining; Post (black), post-training; Rt (circles), at rest; HET (triangles), immediately after HET; MFI, median fluorescence intensity. ${ }^{*} p<0.05$, Rt vs. HET; $+p<0.05$, pre vs. post. Values are the mean \pm SEM.

\subsection{Adhesion- and Senescence-Related Molecules on Erythrocytes}

No alterations in adhesion molecule expression (CD49d and CD36) on erythrocytes were observed after either acute HET or 6 weeks of intervention (Figure 4A,B). However, an acute bout of HET significantly decreased senescence-related CD47 and CD147 expression (Figure 4C,D), which demonstrated enhanced erythrocyte senescence. HE further downregulated CD47 and CD147 expression, even at rest. Unlike HE, NE did not change the levels of CD47 and CD147 after 6 weeks.
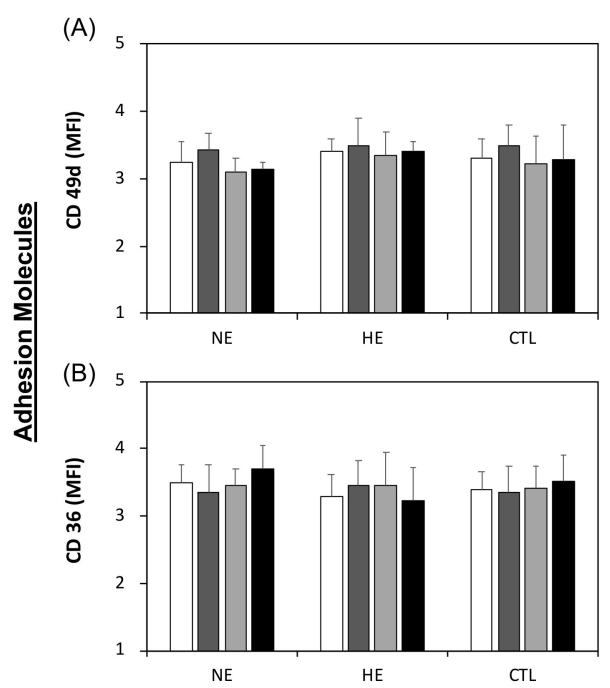
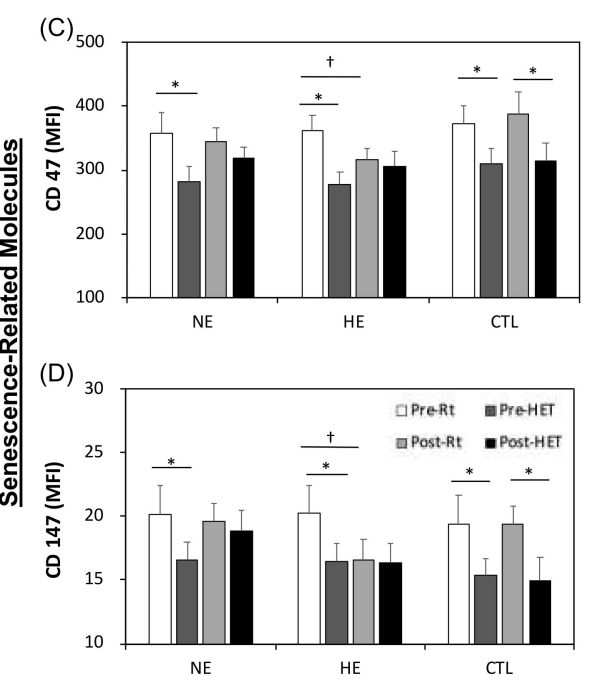

Figure 4. Measurement of adhesion- and senescence-related biomarkers in erythrocytes. The median fluorescence intensities of (A) CD49d, (B) CD36, (C) CD47, and (D) CD147. NE, normoxia training group; HE, hypoxia training group; CTL, control group; Pre, pretraining; Post, post-training; Rt, at rest; HET, immediately after HET; MFI, median fluorescence intensity. ${ }^{*} p<0.05$, Rt vs. HET; $+p<0.05$, pre vs. post. Values are the mean \pm SEM.

\subsection{The Relative Erythrocyte Subfractions}

As verified by flow cytometry analysis, the subfraction of erythrocytes changed (Figure 5). Although acute HET resulted in an increase in the reticulocyte percentage (Figure 5A), it did not influence the mature percentage. After 6 weeks of HE, significantly increased reticulocyte and stress reticulocyte percentages (Figure 5B), coupled with reduced erythrocyte maturation (Figure 5C), indicated accelerated cell turnover under stress. 
(A)

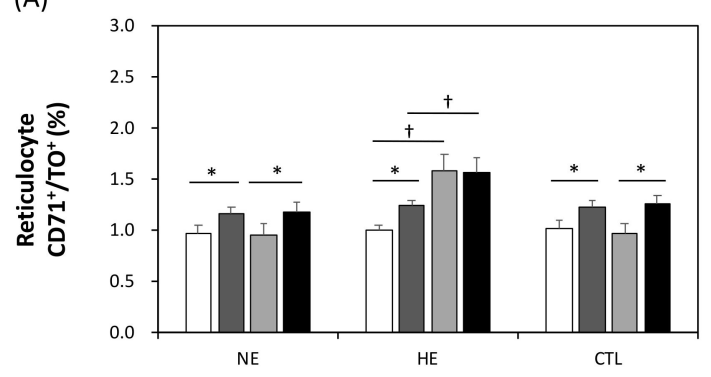

(B)

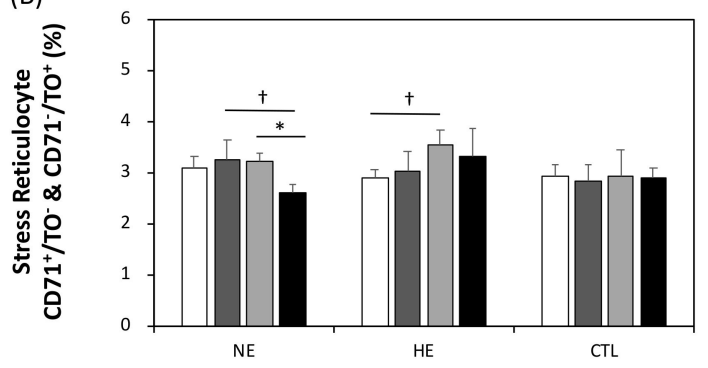

(C)

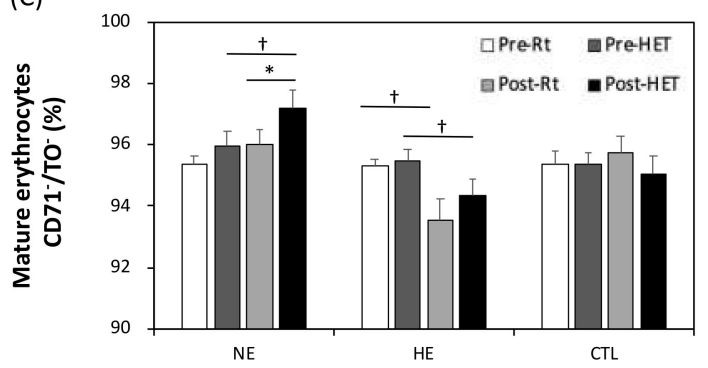

Figure 5. Measurement of erythrocyte composition: (A) the percentage of reticulocytes, (B) the percentage of stress reticulocytes, and $(\mathbf{C})$ the percentage of mature erythrocytes. NE, normoxia training group; HE, hypoxia training group; CTL, control group; Pre, pretraining; Post, post-training; $\mathrm{Rt}$, at rest; HET, immediately after HET; ${ }^{*} p<0.05$, Rt vs. HET; $+p<0.05$, pre vs. post. Values are the mean \pm SEM.

\section{Discussion}

\subsection{Main Discussion}

Erythrocyte aggregation is an intrinsic property of native blood that has a direct effect on blood viscosity and circulation, especially at low shear stress. The aggregation of erythrocytes is determined by intrinsic (e.g., cell surface charge) and extrinsic (e.g., plasma fibrinogen concentration, hematocrit, shear rate) factors [26]. This study first demonstrated that an acute $12 \%$ oxygen hypoxia exercise test augmented erythrocyte aggregation, which was mainly mediated by the intrinsic factors of erythrocytes. Furthermore, HE had a superior effect to NE in ameliorating cardiopulmonary capacity. After 6 weeks of interventions, only the HE further promoted aggregation via both intrinsic aggregability and fibrinogen-erythrocyte interaction, which was accomplished with accumulating counts of stress reticulocytes and erythrocyte senescence. We further demonstrated that NE combined with either decreased fibrinogen binding factor or increased intrinsic aggregability, which seems to be the rationale as to why NE did not alter the aggregation reaction after 6 weeks.

Hemoconcentration is one of the most considerable factors in increasing blood aggregation [27], which increases the opportunity for cell-to-cell contact and largely explains the elevated aggregation during exercise [28]. However, aggregation was not different for suspensions with standardized hematocrit, indicating that hemoconcentration was less relevant to the increased aggregation caused by an acute HET. In contrast to the acute effect, 
hemodilution, a usual long-term effect of regular exercise, was not found in either HE or $\mathrm{NE}$; erythrocyte and hemoglobin counts and hematocrit levels were not apparently altered with either exercise regimen.

Hypoxia and physical exercise are independent of each other and are both highly potent metabolic stressors. Combined, these factors significantly elevated the degree of whole blood aggregation, as we noticed after acute HET and HE. Hypoxia can stimulate EPO secretion under the regulation of HIF-1 [15]. However, a severe hypoxic condition or acute exercise may enhance proinflammatory factor levels, such as those of MPO, TNF- $\alpha$, IL-1, or IL-6. This status may inhibit the EPO response and hematological adaptations [29]. With inflammation, an elevated fibrinogen concentration is mainly responsible for enhancing aggregation [7]. In the clinical setting, a patient with exertional periodic breathing resembles the model of intermittent hypoxia exposure, likely triggering increased ROS [30] and proinflammatory status [31], thus further deteriorating erythrocyte aggregation [32]. Interestingly, neither normoxic exercise training alone nor long-term hypoxic exposure alone was sufficient to suppress those proinflammatory cytokine effects [33]. However, the $15 \%$ oxygen exercise training used in the present study has been demonstrated to suppress MPO and IL-6 production and lipid peroxidation caused by severe exercise or acute hypoxia [34]. Although the plasma fibrinogen concentration responded to the $15 \%$ oxygen exercise training, further investigation is still needed.

In contrast to the NE, the HE dramatically increased ROS during hypoxic training, presumed to further lead to CD47 and CD147 downregulation and enhanced erythrocyte aggregation [35]. Elevated ROS levels are speculated to stabilize HIF-1 production [36]. We previously pointed out that HE can strongly deteriorate erythrocyte antioxidative capacity [13]. Upon return to normoxia, there was an excessive accumulation of ROS in reticulocyte mitochondrial mass [37]. Although possible changes in ROS parameters were not investigated in the present study, due to the above information, it can be speculated here that the impaired hemorheology herein may at least partly be due to increased ROS.

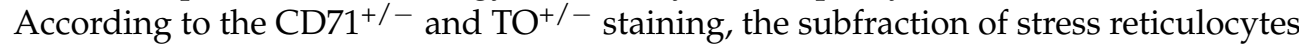
significantly increased after HE [16]. During erythropoietic stress, reticulocytes exit early from the hematopoietic tissues, increasing circulating reticulocyte numbers. Although this staining approach could not distinguish the relative amounts of very old erythrocytes, the levels of CD47 and CD147 showed a significant aging process in the HE. In contrast, the NE increased the relative proportion of mature erythrocytes significantly and concomitantly suppressed senescence. This has been speculated as a relatively positive effect on accelerating erythrocyte maturation due to lower ROS production within 6 weeks of NE [38].

One of the main factors in erythrocyte aggregation is the surface charge; we speculate that the change in membrane sialic acid with erythrocyte aging or even maturation is mainly responsible for training adaptations [39]. Therefore, both NE and HE partly facilitated aggregation via the intrinsic factor. In this work, the aggregation of erythrocytes in the dextran solution reflects intrinsic erythrocyte aggregability [40]. Donor illness or old erythrocytes reduce the surface charge density of erythrocytes, thus increasing aggregation in standard dextran solution [41]. Certainly, there are other age-related cellular factors that influence aggregation, since differences between younger and older cells still exist after removing sialic acid from the erythrocyte surface.

We demonstrated that, in the presence of fixed fibrinogen concentrations, only HE erythrocytes tended to increase in fibrinogen binding probability and force, whereas NE diminished this response. Conversely, some studies have indicated that the fibrinogen binding probability is decreased upon erythrocyte aging due to progressive impairment of the specific fibrinogen-erythrocyte receptor interaction [5]. In addition to the enhanced presence of PS exposure on erythrocytes with aging following the HE, the increased percentage of reticulocytes may also be a contributor to the increased fibrinogen affinity [42]. The reduced binding probability after the NE was probably related to the glycoprotein 
receptors IIb/IIIa (GPIIb/IIIa). Moderate exercise $\left(60 \% \dot{\mathrm{VO}}_{2 \max }\right.$ for $\left.40 \mathrm{~min}\right)$ had a positive influence on GPIIb/IIIa receptor expression, decreasing its baseline percentage values [43].

The beneficial or detrimental effects of intermittent hypoxia may vary substantially with the concentration of oxygen in the air and the subsequent change in the circulatory redox status under an exercise regimen. Although both $12 \%$ oxygen and $15 \%$ oxygen for 8 weeks improved the aerobic fitness of subjects by enhancing pulmonary ventilation and tissue oxygen utilization, our team demonstrated that $12 \%$ oxygen but not $15 \%$ oxygen acute exercise simultaneously enhanced oxidative-stress-induced lymphocyte apoptosis by diminishing cellular antioxidant levels [34]. Therefore, in the present study, the oxygen concentration of the HET was set to $12 \%$. Moreover, chronic intermittent exposure to $12 \%$ oxygen for $1 \mathrm{~h} /$ day for 8 weeks increased levels of proinflammatory cytokines IL-6 and IL-10 and reduced antioxidative capacity [17]. In contrast to the $12 \%$ oxygen, the $15 \%$ oxygen moderate hypoxic exercise training increases resistance to disturbance of cardiac hemodynamics by severe hypoxia [11] and further depresses oxidative stress and proinflammatory cytokine production [33]. Taken together, our numerous previous experimental findings helped us to determine effective hypoxic exercise regimens under $15 \%$ oxygen instead of $12 \%$ to increase aerobic capacity and minimize the risk of inflammatory and thrombotic disorders associated with exercise.

\subsection{Clinical Prospects}

Chronic hypoxic exercise may provide preconditional acclimatization [44] for patients with hypoxia-related disease but also elevate the hypoxia risk of increasing erythrocyte aggregation probability. The $\mathrm{SpO}_{2}$ was around $92 \%$ at $15 \%$ oxygen $(2733 \mathrm{~m})$ in healthy and young subjects (data not shown), whereas COPD patients showed further reduced $\mathrm{SpO}_{2}$ to around $85 \%$ at $2590 \mathrm{~m} \mathrm{[45],} \mathrm{and} \mathrm{the} \mathrm{oxygenation} \mathrm{may} \mathrm{worsen} \mathrm{even} \mathrm{with} \mathrm{a} \mathrm{minimal} \mathrm{level}$ of exertion [46]. Thus, the detrimental effects induced by $15 \%$ oxygen hypoxic exercise in healthy and young men may be worse in chronic lung disease patients.

Moreover, many patients with COPD suffer from intermittent hypoxia during their daily life. In COPD daily life, the mean $\mathrm{SaO}_{2}$ is $88 \%$ and the number of desaturations is 13.1 times $\mathrm{h}^{-1}$ during daily walking, which is a significant deterioration, compared to that during resting [47]. Therefore, even in daily life, physical activity under a hypoxic status may make these patients susceptible to hypoxia-related adverse health effects, thus further elevating erythrocyte aggregation. Interestingly, aside from increased dyspnea, no severe adverse events were reported for exercise under hypoxia, although in several of these studies, many patients were unable to complete the $15 \%$ oxygen exercise task [48]. Aside from the influence of intermittent hypoxia, increases in the levels of IL-6, IL-8, and fibrinogen were demonstrated in COPD [49]. Therefore, our experimental findings facilitate the identification of effective hypoxic exercise regimens for improving aerobic fitness and simultaneously avoiding erythrocyte aggregation [50] to minimize hypoxicinduced rheological deterioration.

\subsection{Study Limitations}

The small sample size ( $n=20$ in each group) was a major limitation of this study. However, the results for cardiopulmonary capacity and rheological properties obtained from this investigation have high statistical power (0.846 to 1.000). This study did not directly measure the changes in plasma fibrinogen and reactive oxygen species caused by NE or HE intervention but instead indirectly measured the fibrinogen binding force and probability of erythrocytes. Therefore, the changes in plasma fibrinogen and reactive oxygen species modulated by normoxic and hypoxic exercise regimens must be evaluated further. Clinical investigations have shown that increased erythrocyte aggregation is closely correlated with various circulatory diseases, such as myocardial ischemia and infarction [1], cerebral infarction [2], and diabetes mellitus [3]. However, the subjects recruited in this study were young and healthy; hence, further clinical evidence is required to extrapolate the present results to patients with hemorheological or hemodynamic disorders. The 
study did not detect the levels of haptoglobin, bilirubin, and ferritin, which serve as indirect markers of hemolysis and hemoglobin breakdown, while markers of progressing erythrocyte senescence appear even on reticulocytes; these require further investigation.

\section{Conclusions}

An acute bout of exercise in the air with $12 \%$ oxygen enhanced erythrocyte aggregation and facilitated erythrocyte senescence in sedentary men. After 6 weeks of the intervention, $\mathrm{HE}$ was superior to NE in ameliorating cardiopulmonary capacity; however, HE also resulted in advanced and deteriorated rheological aggregation. Moreover, this augmented aggregation is related to both intrinsic and extrinsic factors, especially the affinity to fibrinogen (Figure 6). Therefore, chronic hypoxic exercise may provide preconditional acclimatization for patients with hypoxia-related disease. We should establish a safe and effective hypoxic exercise prescription in the future.

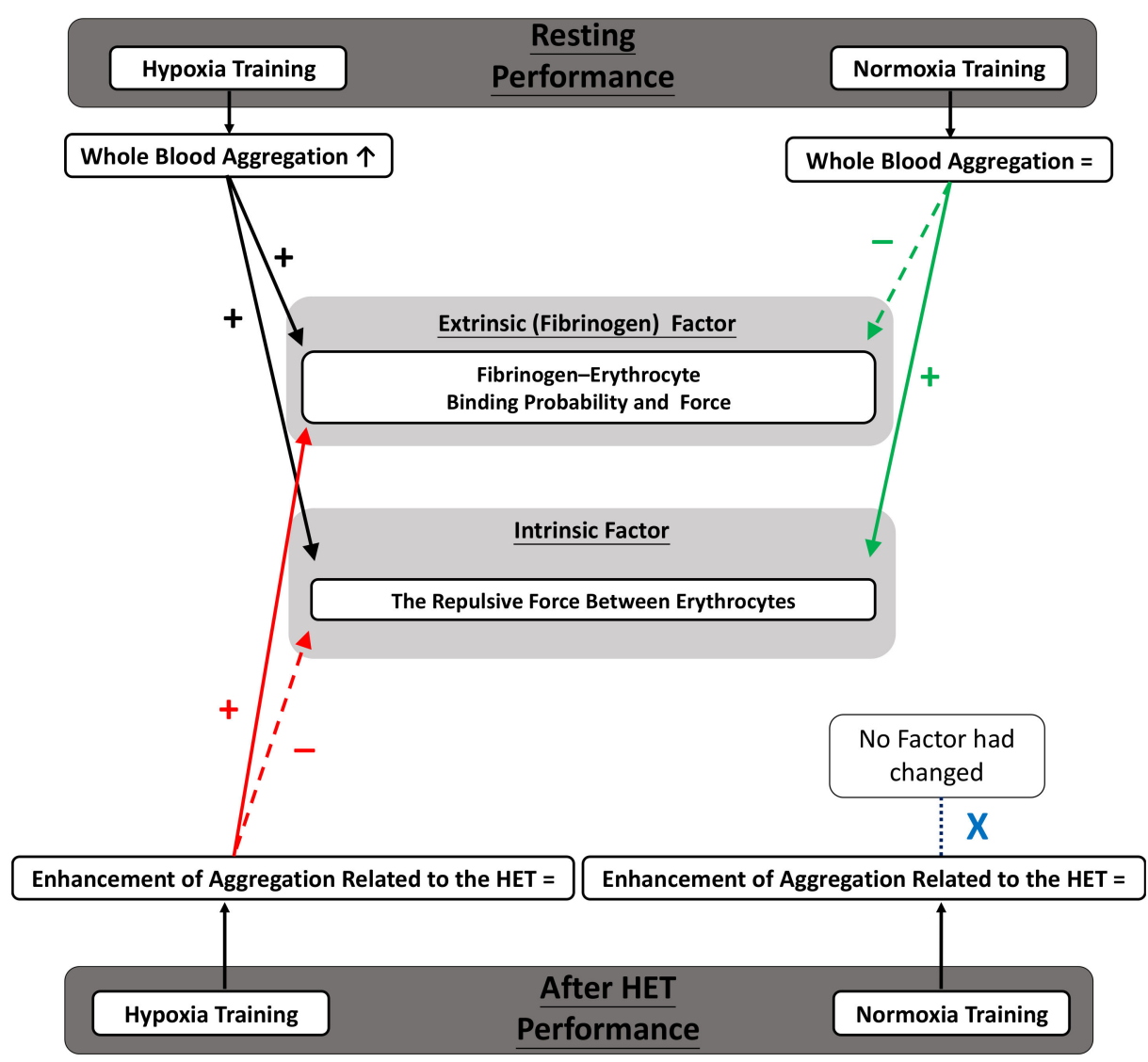

Figure 6. Possible mechanism of the present study. After training, the HET-related aggregation level was not altered in either group; however, after $\mathrm{HE}$, the resting aggregation was significantly augmented via enhancements in both intrinsic and extrinsic response. In contrast to HE, NE elevated the intrinsic aggregability but also diminished the affinity between erythrocytes and fibrinogen. Solid line: enhanced effect; Dashed line: diminished effects.

Author Contributions: Conceptualization, J.-S.W. and Y.-C.H.; methodology, C.-L.L., J.-S.W. and Y.-C.H.; formal analysis, C.-L.L. and J.-S.W.; investigation, C.-L.L. and J.-S.W.; writing-original draft preparation, Y.-C.H.; writing-review and editing, Y.-C.H., C.-L.L., J.-S.W., T.-C.F. and C.-C.H.; supervision, Y.-C.H.; funding acquisition, J.-S.W. and Y.-C.H. All authors have read and agreed to the published version of the manuscript.

Funding: This research was funded by the Ministry of Science and Technology, Grant Number MOST 109-2314-B-468-004, and by the Chang Gung Medical Research Program, Grant Number 
CMRPD1J0222. The APC was funded by Healthy Aging Research Center, Chang Gung University supported from the Ministry of Education in Taiwan, Grant Number EMRPD1L0371.

Institutional Review Board Statement: The study was conducted according to the guidelines of the Declaration of Helsinki and approved by the Institutional Review Board of Chang Gung Memorial Hospital (protocol code 200903510B0).

Informed Consent Statement: Written informed consent was obtained from the patients to publish this paper.

Data Availability Statement: All data is contained within the article.

Acknowledgments: The authors would like to thank the volunteers for their enthusiastic participation and the medical writers, proofreaders, and editors for their assistance.

Conflicts of Interest: The authors declare no conflict of interest. The funders had no role in the design of the study; in the collection, analyses, or interpretation of data; in the writing of the manuscript; or in the decision to publish the results.

\section{References}

1. Saldanha, C.; P Lopes de Almeida, J. Erythrocyte as a link between basic and clinical research. Clin. Hemorheol. Microcirc. 2011, 49, 463-472. [CrossRef]

2. Tikhomirova, I.A.; Oslyakova, A.O.; Mikhailova, S.G. Microcirculation and blood rheology in patients with cerebrovascular disorders. Clin. Hemorheol. Microcirc. 2011, 49, 295-305. [CrossRef]

3. Toker, S.; Rogowski, O.; Melamed, S.; Shirom, A.; Shapira, I.; Berliner, S.; Zeltser, D. Association of components of the metabolic syndrome with the appearance of aggregated red blood cells in the peripheral blood. An unfavorable hemorheological finding. Diabetes/Metab. Res. Rev. 2005, 21, 197-202. [CrossRef]

4. Lipowsky, H.H. Microvascular rheology and hemodynamics. Microcirculation 2005, 12, 5-15. [CrossRef] [PubMed]

5. Carvalho, F.A.; de Oliveira, S.; Freitas, T.; Gonçalves, S.; Santos, N.C. Variations on fibrinogen-erythrocyte interactions during cell aging. PLoS ONE 2011, 6, e18167. [CrossRef]

6. Hierso, R.; Waltz, X.; Mora, P.; Romana, M.; Lemonne, N.; Connes, P.; Hardy-Dessources, M.D. Effects of oxidative stress on red blood cell rheology in sickle cell patients. Br. J. Haematol. 2014, 166, 601-606. [CrossRef] [PubMed]

7. Sewchand, L.S.; Canham, P.B. Modes of rouleaux formation of human red blood cells in polyvinylpyrrolidone and dextran solutions. Can. J. Physiol. Pharmacol. 1979, 57, 1213-1222. [CrossRef]

8. Deng, L.H.; Barbenel, J.C.; Lowe, G.D. Influence of hematocrit on erythrocyte aggregation kinetics for suspensions of red blood cells in autologous plasma. Biorheology 1994, 31, 193-205. [CrossRef]

9. Huang, Y.C.; Hsu, C.C.; Wang, J.S. High-Intensity Interval Training Improves Erythrocyte Osmotic Deformability. Med. Sci. Sports Exerc. 2019, 51, 1404-1412. [CrossRef] [PubMed]

10. Raynaud, E.; Perez-Martin, A.; Brun, J.; Aïssa-Benhaddad, A.; Fédou, C.; Mercier, J. Relationships between fibrinogen and insulin resistance. Atherosclerosis 2000, 150, 365-370. [CrossRef]

11. Wang, J.S.; Wu, M.H.; Mao, T.Y.; Fu, T.C.; Hsu, C.C. Effects of normoxic and hypoxic exercise regimens on cardiac, muscular, and cerebral hemodynamics suppressed by severe hypoxia in humans. J. Appl. Physiol. 2010, 109, 219-229. [CrossRef] [PubMed]

12. Grygorczyk, R.; Orlov, S.N. Effects of Hypoxia on Erythrocyte Membrane Properties-Implications for Intravascular Hemolysis and Purinergic Control of Blood Flow. Front. Physiol. 2017, 8, 1110. [CrossRef] [PubMed]

13. Mao, T.Y.; Fu, L.L.; Wang, J.S. Hypoxic exercise training causes erythrocyte senescence and rheological dysfunction by depressed Gardos channel activity. J. Appl. Physiol. 2011, 111, 382-391. [CrossRef]

14. Vij, A.G. Effect of prolonged stay at high altitude on platelet aggregation and fibrinogen levels. Platelets 2009, $20,421-427$. [CrossRef]

15. Semenza, G.L.; Shimoda, L.A.; Prabhakar, N.R. Regulation of gene expression by HIF-1. Novartis Found. Symp. 2006, $272,2-36$.

16. Rhodes, M.M.; Koury, S.T.; Kopsombut, P.; Alford, C.E.; Price, J.O.; Koury, M.J. Stress reticulocytes lose transferrin receptors by an extrinsic process involving spleen and macrophages. Am. J. Hematol. 2016, 91, 875-882. [CrossRef]

17. Wang, J.S.; Lin, H.Y.; Cheng, M.L.; Wong, M.K. Chronic intermittent hypoxia modulates eosinophil- and neutrophil-platelet aggregation and inflammatory cytokine secretion caused by strenuous exercise in men. J. Appl. Physiol. 2007, 103, 305-314. [CrossRef]

18. Pialoux, V.; Hanly, P.J.; Foster, G.E.; Brugniaux, J.V.; Beaudin, A.E.; Hartmann, S.E.; Pun, M.; Duggan, C.T.; Poulin, M.J. Effects of exposure to intermittent hypoxia on oxidative stress and acute hypoxic ventilatory response in humans. Am. J. Respir. Crit. Care Med. 2009, 180, 1002-1009. [CrossRef]

19. Fu, Q.; Townsend, N.E.; Shiller, S.M.; Martini, E.R.; Okazaki, K.; Shibata, S.; Truijens, M.J.; Rodríguez, F.A.; Gore, C.J.; StrayGundersen, J.; et al. Intermittent hypobaric hypoxia exposure does not cause sustained alterations in autonomic control of blood pressure in young athletes. Am. J. Physiol. Regul. Integr. Comp. Physiol. 2007, 292, R1977-R1984. [CrossRef] 
20. Lee, P.H.; Macfarlane, D.J.; Lam, T.H.; Stewart, S.M. Validity of the International Physical Activity Questionnaire Short Form (IPAQ-SF): A systematic review. Int. J. Behav. Nutr. Phys. Act. 2011, 8, 115. [CrossRef]

21. Ortega, R.M.; Perez-Rodrigo, C.; Lopez-Sobaler, A.M. Dietary assessment methods: Dietary records. Nutr. Hosp. 2015,31 (Suppl. 3), 38-45. [CrossRef] [PubMed]

22. Thompson, W.R.; Hubertus, G.M.; Pescatello, L.S. Clinical Exercise Testing. In ACSM's Guidelines for Exercise Testing and Prescription; American College of Sports Medicine: Indianapolis, IN, USA, 2017.

23. Chen, Y.C.; Ho, C.W.; Tsai, H.H.; Wang, J.S. Interval and continuous exercise regimens suppress neutrophil-derived microparticle formation and neutrophil-promoted thrombin generation under hypoxic stress. Clin. Sci. 2015, 128, 425-436. [CrossRef]

24. Samsel, L.; McCoy, J.P., Jr. Imaging flow cytometry for the study of erythroid cell biology and pathology. J. Immunol. Methods 2015, 423, 52-59. [CrossRef]

25. Lee, L.G.; Chen, C.H.; Chiu, L.A. Thiazole orange: A new dye for reticulocyte analysis. Cytometry 1986, 7, 508-517. [CrossRef]

26. Baskurt, O.; Meiselman, H. Blood Rheology and Hemodynamics. Semin. Thromb. Hemost. 2003, 29, 435-450. [CrossRef]

27. Brun, J.F.; Connes, P.; Varlet-Marie, E. Alterations of blood rheology during and after exercise are both consequences and modifiers of body's adaptation to muscular activity. Sci. Sports 2007, 22, 251-266. [CrossRef]

28. Mairbäurl, H. Red blood cells in sports: Effects of exercise and training on oxygen supply by red blood cells. Front. Physiol. 2013, 4, 332. [CrossRef]

29. Morceau, F.; Dicato, M.; Diederich, M. Pro-Inflammatory Cytokine-Mediated Anemia: Regarding Molecular Mechanisms of Erythropoiesis. Mediat. Inflamm. 2009, 405016. [CrossRef]

30. Prabhakar, N.R.; Kumar, G.K.; Nanduri, J.; Semenza, G.L. ROS Signaling in Systemic and Cellular Responses to Chronic Intermittent Hypoxia. Antioxid. Redox Signal. 2007, 9, 1397-1404. [CrossRef] [PubMed]

31. Dyugovskaya, L.; Polyakov, A.; Ginsberg, D.; Lavie, P.; Lavie, L. Molecular pathways of spontaneous and TNF- $\alpha-m e d i a t e d$ neutrophil apoptosis under intermittent hypoxia. Am. J. Respir. Cell Mol. Biol. 2011, 45, 154-162. [CrossRef]

32. Wang, J.S.; Fu, T.C.; Wang, C.H.; Chou, S.L.; Liu, M.H.; Cherng, W.J. Exertional periodic breathing potentiates erythrocyte rheological dysfunction by elevating pro-inflammatory status in patients with anemic heart failure. Int. J. Cardiol. 2013, 167, 1289-1297. [CrossRef]

33. Wang, J.-S.; Chen, W.-L.; Weng, T.-P. Hypoxic exercise training reduces senescent T-lymphocyte subsets in blood. BrainBehav. Immun. 2011, 25, 270-278. [CrossRef]

34. Wang, J.S.; Lin, C.T. Systemic hypoxia promotes lymphocyte apoptosis induced by oxidative stress during moderate exercise. Eur. J. Appl. Physiol. 2010, 108, 371-382. [CrossRef] [PubMed]

35. Varlet-Marie, E.; Brun, J.F. Reciprocal relationships between blood lactate and hemorheology in athletes: Another hemorheologic paradox? Clin. Hemorheol. Microcirc. 2004, 30, 331-337.

36. Guzy, R.D.; Hoyos, B.; Robin, E.; Chen, H.; Liu, L.; Mansfield, K.D.; Simon, M.C.; Hammerling, U.; Schumacker, P.T. Mitochondrial complex III is required for hypoxia-induced ROS production and cellular oxygen sensing. Cell Metab. 2005, 1, 401-408. [CrossRef] [PubMed]

37. Song, J.; Yoon, D.; Christensen, R.D.; Horvathova, M.; Thiagarajan, P.; Prchal, J.T. HIF-mediated increased ROS from reduced mitophagy and decreased catalase causes neocytolysis. J. Mol. Med. 2015, 93, 857-866. [CrossRef]

38. Ventura, N.; Hoppeler, H.; Seiler, R.; Binggeli, A.; Mullis, P.-E.; Vogt, M. The Response of Trained Athletes to Six Weeks of Endurance Training in Hypoxia or Normoxia. Int. J. Sports Med. 2003, 24, 166-172. [CrossRef]

39. Huang, Y.X.; Wu, Z.J.; Mehrishi, J.; Huang, B.T.; Chen, X.Y.; Zheng, X.J.; Liu, W.J.; Luo, M. Human red blood cell aging: Correlative changes in surface charge and cell properties. J. Cell. Mol. Med. 2011, 15, 2634-2642. [CrossRef]

40. Baskurt, O.N.; Neu., B.; Meiselman, H.J. Red Blood Cell Aggregation, 1st ed.; CRC Press: Boca Raton, FL, USA, $2012 ;$ pp. 31-59.

41. Neu, B.; Sowemimo-Coker, S.O.; Meiselman, H.J. Cell-cell affinity of senescent human erythrocytes. Biophys. J. 2003, 85, 75-84. [CrossRef]

42. De Oliveira, S.; Vitorino de Almeida, V.; Calado, A.; Rosário, H.S.; Saldanha, C. Integrin-associated protein (CD47) is a putative mediator for soluble fibrinogen interaction with human red blood cells membrane. Biochim. Et Biophys. Acta 2012, 1818, 481-490. [CrossRef] [PubMed]

43. Fiodorenko-Dumas, Ż.; Dumas, I.; Mastej, K.; Jakobsche-Policht, U.; Bittner, J.; Adamiec, R. Receptor GP IIb/IIIa as an Indicator of Risk in Vascular Events. Clin. Appl. Thromb. Hemost. 2019, 25, 1076029619845056. [CrossRef]

44. Viscor, G.; Torrella, J.R.; Corral, L.; Ricart, A.; Javierre, C.; Pages, T.; Ventura, J.L. Physiological and Biological Responses to Short-Term Intermittent Hypobaric Hypoxia Exposure: From Sports and Mountain Medicine to New Biomedical Applications. Front. Physiol. 2018, 9, 814. [CrossRef]

45. Latshang, T.D.; Tardent, R.P.M.; Furian, M.; Flueck, D.; Segitz, S.D.; Mueller-Mottet, S.; Kohler, M.; Ulrich, S.; Bloch, K.E. Sleep and breathing disturbances in patients with chronic obstructive pulmonary disease traveling to altitude: A randomized trial. Sleep 2019, 42. [CrossRef]

46. Mestry, N.; Thirumaran, M.; Tuggey, J.M.; Macdonald, W.; Elliott, M.W. Hypoxic challenge flight assessments in patients with severe chest wall deformity or neuromuscular disease at risk for nocturnal hypoventilation. Thorax 2009, 64, 532-534. [CrossRef]

47. Soguel Schenkel, N.; Burdet, L.; de Muralt, B.; Fitting, J.W. Oxygen saturation during daily activities in chronic obstructive pulmonary disease. Eur. Respir. J. 1996, 9, 2584-2589. [CrossRef] 
48. Seccombe, L.M.; Kelly, P.T.; Wong, C.K.; Rogers, P.G.; Lim, S.; Peters, M.J. Effect of simulated commercial flight on oxygenation in patients with interstitial lung disease and chronic obstructive pulmonary disease. Thorax 2004, 59, 966-970. [CrossRef] [PubMed]

49. Verges, S.; Chacaroun, S.; Godin-Ribuot, D.; Baillieul, S. Hypoxic Conditioning as a New Therapeutic Modality. Front. Pediatrics 2015, 3, 58. [CrossRef] [PubMed]

50. Ugurlu, E.; Kilic-Toprak, E.; Can, I.; Kilic-Erkek, O.; Altinisik, G.; Bor-Kucukatay, M. Impaired Hemorheology in Exacerbations of COPD. Can. Respir. J. 2017, 2017, 1286263. [CrossRef] 